African Journal for Physical, Health Education, Recreation and Dance (AJPHERD)

Vol. 13, No. 3 (September) 2007, pp. 267-273.

\section{EFFECTS OF EXERCISE ON THE VISUAL PERFORMANCE OF FEMALE RUGBY PLAYERS}

\section{P.J. du Toit, P.E. Krüger, A. Joubert and J. Lunsky}

\begin{abstract}
Visual performance is an important factor in sport excellence. Over the past few years, aspects like hand-eye co-ordination, visual reaction time and their relation to eye exercises have been addressed frequently. Theories maintain that visual involvement varies according to the environmental demands, and that athlete's visual characteristics therefore vary according to the sports in which they specialise, have also been covered. It is well known that environmental demands are matched by task-specific motor response. The extension of this theory shows that visual ability can affect both motor learning and performance, and that the nature of the visual involvement will vary according to environmental demands. The purpose of this study was to determine if exercise will improve the visual performance of female rugby players, in order to achieve maximal results on the sports field. Twenty female rugby players aged 19 to 24 were chosen as subjects. In order to evaluate the effect of sport vision specific exercises on hand-eye co-ordination, a pretest post-test experimental groups design was adopted for the study. The pre-exercise and post-exercise values of the subjects in the control and experimental groups were pooled to determine the averages and differences. From the results, it can be seen that there was an improvement in the performance of the subjects who were exposed to the specific exercises. This study could assist in advocating a theoretical framework and providing an empirical data base to guide and evaluate future research.
\end{abstract}

Key words: Visual performance, exercise, female rugby players.

P.J. du Toit, $P h D$ (Physiology)

Department of Human Physiology.

P.E. Krüger, DPhil. (Physical Education)

A. Joubert

J. Lunsky

Department of Biokinetics, Sport and Leisure

Sciences, University of Pretoria, South Africa

\section{INTRODUCTION}

Visual performance is an important factor in excellence in sport (Haywood, 1984; Granet, 1988; Sale, 1998), and most sport activities are performed less effectively with poor vision (Wood, Woods \& Jack, 1994). Visual ability can affect both motor learning and performance, and the nature of the visual involvement will vary according to environmental demands (Du Toit, Lunsky, Van Vuuren, Van Heerden, Kruger \& Steinmann, 1999).

One of the areas requiring study is the effect of specific exercises on hand-eye co-ordination. Evidence concerning the relationship between exercise and human information processing differs, suggesting a positive relationship (Tilley \& Bohle, 1988; Brisswalter \& Legros, 1996), no relationship (Tomporowski, Ellis \& Stephens, 1987), a curvilinear relationship (Reilly \& Smith, 1986) and a negative relationship (Fleury \& Bard, 1987). Hand-eye co-ordination is the ability of the eyes, hands and body to work as one division to ensure effective response to visual stimuli (Du Toit, Krüger, De Wet, Van Vuuren, Joubert, Lottering \& Van Wyk, 2006).

E-mail: ernst.kruger@up.ac.za 
Co-ordination occurs when the motor system composes complex actions by combining simpler sub-movements. The process involves sharing information about the progress of one sub-movement with the centres controlling another sub-movement, to ensure that the second happens in an appropriate relation to the first (Haggard, 1997). High-quality athletes have the ability to concentrate and stay focused for long periods of time (Haggard, 1997). Hard work, dedication and correct training will help the athlete in the game and maintain his or her focus. Investigating the effect of specific exercises on hand-eye coordination of female rugby players is obviously important due to the fact that:

most sports involving projectiles (balls) and the use of extended levers (bats), involve simultaneous strenuous physical activity and superior hand-eye co-ordination; and

athletes will be enabled to take full advantage of the potential of sports vision and vision training (Hazel, 1995).

The purpose of this study was to determine if specific exercises will improve the visual performance of female rugby players, in order to achieve maximal results on the sports field.

\section{MATERIALS AND METHODS}

Twenty female rugby players aged 19 to 24 years were chosen as subjects. In order to evaluate the effect of physical exercise on hand-eye co-ordination, a pretest - post-test experimental groups design was adopted for the study. All players were subjected to a repeated measures protocol, using a Sport Vision Battery consisting of five different hand-eye co-ordination tests. The experimental group members $(\mathrm{n}=10)$ were exposed to a ten-minute period of isolated exercises as a physical intervention between the pretests and post-tests of the five different hand-eye co-ordination tests, while the controls $(n=10)$ remained inactive during the period between the initial test and the retest, after which the same sports vision test was repeated and the results compared. An easy and reliable method for determining effort during running is to monitor the heart rate during exercise (Noakes \& Granger, 1995). Heart rate monitoring is important for assessing the total level and pattern of energy expenditure and physical activity in 
medium size and larger scale letters, the time is recorded. The subject epidemiological studies (Wareham, is sitting on a gym ball, introducing a Hennings, Prentice \& Day, 1997). A proprioceptive concentration, resulting general aerobic training intensity of $82 \%$ of maximal heart rate was used on the rugby players, thus making possible the setting of clear, precise, observable limits on physical activity, and selfin the visual system being tested dynamically. The parameters tested in this specific test include visual concentration and hand-eye coordination.

controlling of an acceptable level of physical activity (De Wet, Du Toit, Van Vuuren, Rossouw \& Steinmann, 1999; Bar-Mor, Zeevi, Yaaron \& Falk, 1999). Specialised sports vision testing software (version 1), comprising five specific hand-eye co-ordinated tests, was used to evaluate different parameters of hand-eye co-ordination. The operational definitions, in conjunction with the procedures, instruments and scoring applied in the tests, were as follows:

\section{Rotator Pegboard}

A rotating board with the 26 letters of the alphabet placed randomly around the screen is rotated on the computer screen at a specific constant speed. The subject, using the mouse, has three minutes to click on each letter. The clicking must be done in alphabetical order. Once the subject has successfully clicked on all consecutive

\section{Accuvision 1}

Twenty lights flash randomly on an accuvision board on the computer monitor. As soon as the light on the board is clicked on, the next light will appear. The subject has to complete this as quickly as possible. The parameter tested in this specific test includes proaction-reaction time, i.e. speed of motor reaction time after sensory input.

\section{Accuvision 2}

This consists of 60 lights being flashed at a certain speed and a green light that flashes randomly. If the subject clicks on the flashing light when the green light is not flashing, a negative score is recorded. The score recorded indicates how many lights are clicked on while the green light is on, minus the lights clicked on while the fixation light is off. Accuvision 1 and 2 are performed while the subject is sitting on a gym ball. 
This ball introduces a proprioceptive concentration, resulting in the visual system being tested dynamically. The parameters tested are peripheral awareness, pro-action-reaction time and visual concentration.

\section{Strobespecs}

A ball moves around the monitor at a given speed. At a certain point the ball disappears, allowing the subject limited visual input. The subject must then place a second ball in the anticipated flight direction of the previous ball. The parameter primarily tested is visual anticipation.

\section{Tachitoscope}

A different five-digit number is flashed on the wall 12 times, using a Rheem projector. The first four numbers are flashed at 1/10 second, the next four at $1 / 50$ second and the last four at 1/100 second. The subject writes down each number seen. Scoring is done by allocating points to the answers written down by the subject. If all of the digits are correct they score 1 mark and if 4 or 3 digits are correct (in the correct order) they score $1 / 2$ a mark. The parameters primarily tested are visual response speed, accuracy and short-term visual memory.

\section{Exercises done in between: Simultaneous ball throw out of both hands}

Coach stands $2 \mathrm{~m}$ from subject and throws two balls simultaneously for the subject to catch.

Technical pointers: Bent knees, feet at shoulder width apart. Soft hands bring balls towards body.

Benefits: Improves peripheral vision, concentration and ball handling.

\section{Lateral shuffle and ball catch}

Subject shuffles side to side between cones, $4 \mathrm{~m}$ apart. Coach throws ball to subject at any time. Ball thrown back to coach and subject keeps shuffling.

Technical pointers: Shuffle side to side between the cones, touch the ground at each cone. Look forward, so back is straight. Bend knees to go down or to catch ball. Once ball is caught, continue shuffling from side to side.

Benefits: Improves eye-brain-body coordination and concentration. Improves anaerobic fitness. 


\section{Crossover}

Coach stands $2 \mathrm{~m}$ from subject and throws two balls simultaneously to opposite hands, for the subject to catch. Technical pointers: Bent knees, feet \pm shoulder width apart. Soft hands bring balls towards body.

Benefits: Improves peripheral vision, concentration and ball handling.

\section{2 vs. 1}

Subject is fed with balls by two coaches. One throws a ball to the subject to catch, while shuffling between two cones $4 \mathrm{~m}$ apart.

Technical pointers: Bend knees to go down or catch the ball. Once ball is caught, continue shuffling side to side to the next coach.

\section{Crucifix ball drop}

Coach drops ball for subject to catch, while subject is in a squat position.

Technical pointers: Bent knees, feet at shoulder width apart, and hands on knees. Subject must catch ball with wrist supinated. When ball drops, subject must drop into squat position as quickly as possible. Do not snatch at the ball.

Benefits: Improves peripheral vision. Improves foot speed.

\section{RESULTS AND DISCUSSION}

The pre-exercise and post-exercise values of the subjects in the control and experimental groups were pooled to determine the averages and differences as represented in Table 1.

When the results are studied, it is clear that there was an improvement in the performance of subjects exposed to the physical exercise. Analyses of the data, when comparing post-exercise values of control and experimental groups, showed a significant increase $(\%$ accuracy) with Tachistoscope and an increase (\% accuracy) with the Accuvision 1000 - test $2 \quad[p=0.08]$. Analyses also showed a significant decrease (time taken to complete an activity in seconds) with the Accuvision 1000 - test 1 and Rotator Pegboard.

\section{DISCUSSION}

The athletic ability of a sporting individual depends not only on the cardiopulmonary and metabolic status (Wasserman, 1986; West, 1989) of the individual but also on his or her handeye co-ordination ability. 
Table 1: Pre-exercise and post-exercise (control and experimental groups) average values of the different sports vision tests

\begin{tabular}{|l|l|l|l|l|l|}
\hline \multirow{2}{*}{ Sports vision tests } & Experimental & \multicolumn{4}{l|}{} \\
\cline { 2 - 6 } & Pre-exercise & $\begin{array}{l}\text { Post- } \\
\text { exercise }\end{array}$ & Pre-exercise & $\begin{array}{l}\text { Post- } \\
\text { exercise }\end{array}$ & p \\
\hline Strobespecs (\%) & 37.4 & 34.6 & 44.1 & 42.7 & 0.29 \\
\hline Accuvision - Test 1 (sec) & 21.7 & 20.0 & 19.5 & $17.9^{*}$ & 0.05 \\
\hline Accuvision - Test 2 (\%) & 64.6 & 71.4 & 67.9 & 81.9 & 0.08 \\
\hline Rotator Pegboard (sec) & 43.4 & 41.4 & 39.9 & $33.8^{*}$ & 0.01 \\
\hline Tachistoscope (\%) & 53.5 & 56.0 & 60.0 & $70.0^{*}$ & 0.05 \\
\hline
\end{tabular}

${ }^{*} \mathrm{p}<0.05$ when comparing post-exercise values of control and experimental groups.

The eyes feed information to the brain. If the information is inaccurate, performance may suffer and factors like the eyes' ability to focus clearly, the eyes' ability to quickly and accurately change focus, the eyes' ability to rapidly process visual information, thereby improving reaction time and general eye health, may be affected. The specific exercises used in the experimental group led to a significant improvement in the post-tests, which suggested an improvement in visual concentration, hand-eye co-ordination, proaction-reaction time, visual response speed, accuracy and short-term visual memory. The improvement observed in the control group that was not significant can probably be ascribed to the learning effect of the brain, the subject thus being accustomed to the test. These results confirm the possibility that physical exercise can improve mental performance (Paas \&
Adam, 1991). The results also suggest that hand-eye co-ordination is sensitive to exercise, mediated by factors such as physical fitness levels of subjects; intensity and duration of exercise intervention; co-ordination tests selected by investigator and the time at which the tests were done (Tomporowski \& Ellis, 1986). More factors that could be added after this study is the specific type, task difficulty and intensity of the exercises during the intervention period.

\section{CONCLUSION}

The results suggest that with the correct training programmes and correct handeye co-ordination tests, a vast improvement can be achieved in sporting performance, giving the athlete optimal performance and the advantage above his or her competitors. 


\section{REFERENCES}

Bar-Mor, G., Zeevi, B., Yaaron, M. \& Falk, B. (1999). Use of the heart rate monitor to modulate physical activity in adolescents with congenital aortic stenosis: an innovative approach. Journal of Pediatric Nursing, 14(4), 273-277.

Brisswalter, J. \& Legros, P. (1996). Effect of 3 hours walk race on energy cost and stride rates parameters. International Journal of Sports Medicine, 15, 238-241.

De Wet, K., Du Toit, P.J., Van Vuuren, B., Rossouw J. \& Steinmann, C.M.L. (1999). Determining a general aerobic endurance training intensity for cricket players in the off season by using maximal oxygen uptake, heart rate and blood lactate values as parameters. African Journal for Physical, Health Education, Recreation and Dance, 6(2), 190-203.

Du Toit, P.J., Krüger, P.E., De Wet, K., Van Vuuren, B., Joubert, A., Lottering, M.L. \& Van Wyk, G.J. (2006). Transfer effects of eye-hand co-ordination skills from the right to the left cerebral hemispheres in South African schoolboy rugby players. African Journal for Physical, Health Education, Recreation and Dance, 12(1), 41-49.

Du Toit, P.J., Lunsky, J., Van Vuuren, B., Van Heerden, J., Kruger, M.C. \& Steinmann, C.M.L. (1999). Hand-eye co-ordination of cricket players after a short period of exercise. African Journal for Physical, Health Education, Recreation and Dance, 5(2), 160-168.

Fleury, M. \& Bard, C. (1987). Effects of different types of physical activity on the performance of perceptual tasks in peripheral and central vision and coincident timing. Ergonomics, 30, 945-958.

Granet, D.B. (1988). Objectivity in sports vision testing. International Ophthalmology Clinics, 28, 197-198.

Haggard, P. (1997). Coordinating actions. Quarterly Journal of Experimental Psychology, 50(4), 707-25.

Haywood, K.M. (1984). Use of the imageretina and eye-head movement visual systems during coincidence-anticipation performance. Journal of Sports Science, 2, 139-144.
Hazel, C.A. (1995). The efficacy of sports vision practice and its role in clinical optometry. Clinical and Experimental Optometry; 78, 98105.

Noakes, T. \& Granger, S. (1995). Running Your Best. Oxford: Oxford University Press.

Paas, F.G.W.C. \& Adam, J.J. (1991). Human information processing during physical exercise. Ergonomics, 34, 1385-1397.

Reilly, T. \& Smith, D. (1986). Work intensity and psychomotor performance. Ergonomics, 29, 601-606.

Sale, D.G. (1998). Neural adaptation to resistance training. Medicine and Science in Sports and Exercise, 20, S135.

Tilley, A.J. \& Bohle, P. (1988). Twisting the night away: The effects of all-night disco dancing on reaction time. Perceptual and Motor Skills, 66, 107-112.

Tomporowski, P.D. \& Ellis, N.R. (1986). The effects of exercise on cognitive processes: a review. Psychological Bulletin, 99, 338-346.

Tomporowski, P.D., Ellis, N.R. \& Stephens, R. (1987). The immediate effects of strenuous exercise on free-recall memory. Ergonomics, 30, 121-129.

Wareham, N.J., Hennings, S.J., Prentice, A.M. \& Day, N.E. (1997). Feasibility of heart-rate monitoring to estimate total level and pattern of energy expenditure in a population-based epidemiological study: the Ely Young Cohort Feasibility Study. British Journal of Nutrition, 78(6), 889-900.

Wasserman, K. (1986). A new method for detecting anaerobic threshold by gas exchange. Journal of Applied Physiology, 60, 2020-2027.

West, J.B. (1989). Physiological Basis of Medical Practice. London: Williams and Wilkins, (pp. 729-753).

Wood, J.M., Woods, R.L. \& Jack, M.P. (1994). Exercise does not increase visual field sensitivity. Optometry and Vision Science, 71, 682-684. 